\title{
Identificación de herramientas como soporte en la implementación de la Norma ISO/IEC 29110 (perfil básico)
}

\author{
Jezreel Mejía ${ }^{1}$, Edgar Bonilla $^{1}$, Elizabeth Villanueva ${ }^{1}$, Einar Serna ${ }^{1}$, \\ Israel Faustino ${ }^{1}$, Alessandro Milán ${ }^{1}$. \\ \{jmejia, edgar.bonilla, elizabeth.villanueva, einar.serna, israel.faustino, \\ hector.milan\}@cimat.mx
}

${ }^{1}$ Centro de Investigación en Matemáticas, (CIMAT, A.C.), Unidad Zacatecas, México, Calle Lasec, Andador Galileo Galilei. Andador 3, Lote 7, CP 98160, Zacatecas, México.

DOI: 10.17013/risti.36.15-33

\begin{abstract}
Resumen: Actualmente, se estan dando a conocer normas orientadas a las denominadas Entidades Muy Pequeñas (EMPs) como lo es la norma ISO/IEC 29110. Aun cuando se están creando y dando a conocer nuevas normas orientadas a las EMPs, existe una carencia de herramientas que permitan automatizar o dar soporte en la implementación de estas nuevas normas. Por lo tanto, este articulo, presenta una propuesta de herramientas que den soporte en la implementación a las actividades y tareas de los dos procesos que contiene la norma ISO/IEC 29110.
\end{abstract}

Palabras-clave: ISO/IEC 29110; Herramientas; Gestión de Proyectos; Implementación del Software.

\section{Identification of tools to support ISO/IEC 29110 implementation (basic profile)}

\begin{abstract}
Currently, standards oriented to the so-called Very Small Entities (EMPs) such as the ISO / IEC 29110 standard are becoming known. However, although new standards oriented to EMPs are being created, there is a lack of tools that allow automating or supporting the implementation of these new standards. Therefore, this article presents a tools proposal that support the implementation of the activities and tasks of the two processes of the ISO / IEC 29110 standard.
\end{abstract}

Keywords: ISO/IEC29110; Tools; Project Management; Software Implementation.

\section{Introducción}

En la actualidad, existen en México poco más de 4.1 millones de entidades, de las cuales el $97.3 \%$ son consideradas como micro y el $2.7 \%$ estan dentro del rango de pequeña y mediana esto según datos obtenidos de la encuesta ENAPROSE 2018 llevada a cabo por el INEGI (INEGI, 2020). Las muy pequeñas empresas (VSEs por sus siglas en inglés) 
es una entidad que esta definida como "una empresa”, organización, departamento o proyecto que tiene a su cargo de 1 a 25 personas (ISO/IEC, 2018), (NYCE, 2020). En este contexto, se estan dando a conocer normas orientadas a las denominadas Entidades Muy Pequeñas (EMPs) de desarrollo de software como lo es la norma ISO/IEC 29110. El uso de este tipo de normas en organizaciones de tipo VSEs les ayuda a incremetar su competitivadad, asi como la calidad de sus desarrollos de software (O'Connor, 2017).

La norma ISO/IEC 29110 es un estándar internacional de ingeniería de software que define los perfiles del ciclo de vida para las EMPs que desarrollan software no crítico. Su objetivo, es satisfacer las necesidades específicas de las EMPs y abordar el problema de la baja adopción de normas por parte de estas organizaciones (NYCE. 2020), (ISO/IEC 29110, 2012). Sin embargo, factores como falta de cultura de procesos, conocimiento de normas o metodologias de desarrollo, falta de personal y tiempo para llevar de una manera adecuada la implementacion de esta norma, es complicado para las EMPs implementar de manera adecuda la norma. Ademas, la falta de herramientas que permitan automatizar las actividades o tareas indicadas en las normas es un obstaculo dificil de eliminar y que propicia una barrera para una buena implementacion de este tipo de normas.

Por lo tanto, el objetivo de este trabajo es facilitar una propuesta de implementación de la norma ISO/IEC 29110, proporcionando un ciclo de desarrollo de software con base a los dos procesos Gestión de Proyectos (GP) e Implementación de Software (IS) del perfil básico de esta norma. Además de proponer el uso de herramientas para estos procesos en apoyo a la automatizacion o soporte al registro de informacion o datos que generan o requiere cada tarea y/o actividad de los procesos GP e IS.

El contenido de este documento está estructurado de la siguiente forma: en la sección 2, se presenta una breve introducción acerca de la norma ISO/IEC 29110; en la sección 3, se presenta la propuesta que incluye: el ciclo de desarrollo de software propuesto y las herramientas que dan soporte a las actividades y tareas de los dos procesos GP e IS de esta norma; en la sección 4 muestra el estudio de caso y por último en la sección 5, se muestran las conclusiones y trabajo futuro.

\section{Descripción general del ISO/IEC 29110}

La ISO (Organización Internacional para la Normalización) y la IEC (Comisión Electrotécnica Internacional) forman el sistema especializado para la normalización mundial. Los organismos nacionales miembros de ISO e IEC participan en el desarrollo de las Normas Internacionales por medio de comités técnicos establecidos por la organización respectiva, para atender campos particulares de la actividad técnica (ISO/ IEC ). La ISO/IEC se divide en cinco partes (ISO/IEC, 2018): Parte 1: Visión general, Parte 2: Marco de trabajo y taxonomía, Parte 3: Guía de evaluación, Parte 4: Especificaciones de perfil, Parte 5: Guía de gestión e ingeniería: Provee una guía de implementación sobre gestión e ingeniería para el Perfil Básico del Grupo del Perfil Genérico especificado en la ISO/IEC 29110. La Parte 5-1 (ISO/IEC, 2012) define el Perfil Básico como el desarrollo de software de una sola aplicación por un solo equipo de proyecto sin ningún riesgo especial o factores situacionales. Esta ultima parte, es la utilizada para ser analizada. 


\subsection{Análisis de la estructura de ISO/IEC 29110}

La norma ISO/IEC 29110, está estructurada mediante dos procesos que son Gestión de Proyectos (GP) e Implementación del Software (IS), además, estos cuentan con objetivos $\mathrm{y}$ actividades.

En la Tabla 1, se muestra los roles identificados en la norma ISO/IEC 29110, tanto para GP como para el proceso de Implementación de Software IS.

\begin{tabular}{ll}
\hline Rol & Proceso \\
\hline Cliente (CL) & GP e IS \\
\hline Gestor del Proyecto (GP) & GP e IS \\
\hline Líder Técnico (LT) & GP e IS \\
\hline Equipo de Trabajo (ET) & GP e IS \\
\hline Analista (AN) & IS \\
\hline Diseñador (DIS) & IS \\
\hline Programador (PR) & IS \\
\hline
\end{tabular}

Tabla 1 - Roles en GP e IS.

\subsection{Proceso GP}

PMBOK define la gestión de proyecto de la siguiente manera "es la aplicación de conocimientos, habilidades, herramientas y técnicas para proyectar actividades que cumplan con los requisitos del proyecto" (Project Management Institute, 2013).

El propósito del proceso GP es establecer y llevar a cabo de manera sistemática las tareas del proyecto de implementación de Software, las cuales permiten cumplir con los Objetivos del proyecto en calidad, tiempo y costo esperado (ISO/IEC 29110, 2012).

El proceso GP, a través de la realización de cada una de sus actividades, pretende cumplir 7 objetivos. Ademas, este proceso consta de cuatro actividades y cada una de ellas con un numero especifico de tareas:

- GP.1. Planificación del Proyecto: 15 tareas.

- GP.2. Ejecución del Plan del Proyecto: 6 tareas.

- GP.3. Evaluación y control del Proyecto: 3 tareas.

- GP.4. Cierre del Proyecto: 2 tareas.

En la Tabla 2, se muestra los productos que la ISO/IEC 29110 señala como necesarios para realizar las tareas del proceso de GP, así como también se muestra su origen correspondiente.

\begin{tabular}{ll}
\hline Nombre & Origen \\
\hline Enunciado del Trabajo & $\cdot$ Cliente \\
\hline Configuración del Software & $\cdot$ Implementación de software \\
\hline \multirow{2}{*}{ Solicitud de Cambio } & $\cdot$ Cliente \\
& $\cdot$ Implementación de software \\
\hline
\end{tabular}

Tabla 2 - Productos de entrada GP. 
En la Tabla 3, se muestra los productos que la ISO/IEC 29110 señala como aquellos que se generan al realizar las tareas del proceso de GP, así como también se muestra su destino correspondiente. Los productos internos y los que deben ser guardados en el repositorio del proyecto.

\begin{tabular}{ll}
\hline Nombre & Destino \\
\hline Plan de Proyecto & $\cdot$ Implementación de Software \\
\hline Acta de Aceptación & $\cdot$ Alta Dirección \\
\hline Repositorio del Proyecto & $\cdot$ Implementación de Software \\
\hline Acta de Reunión & $\cdot$ Cliente \\
\hline Configuración de Software & $\cdot$ Cliente \\
\hline
\end{tabular}

Tabla 3 - Productos de salida GP.

\subsection{Proceso IS}

El propósito del proceso de Implementación de Software es la realización sistemática de las actividades de Análisis, Diseño, Construcción, Integración y Pruebas para los productos de Software, nuevos o modificados, de acuerdo con los requisitos especificados ISO/IEC 29110, 2012).

El proceso IS, a través de la realización de cada una de sus actividades, pretende cumplir 7 objetivos, ademas, este proceso de IS consta de seis actividades y cada una de ellas con un numero especifico de tareas:

- IS.1. Inicio de la Implementación de Software: 2 tareas.

- IS.2. Análisis de Requisitos del Software: 7 tareas.

- IS.3. Arquitectura y Diseño Detallado del Software: 8 tareas.

- IS.4. Construcción de Software: 7 tareas.

- IS.5. Integración y Pruebas del Software: 11 tareas.

- $\quad$ IS.6. Entrega del Producto: 8 tareas.

En la Tabla 4, se muestra los productos que la ISO/IEC 29110 señala como necesarios para realizar las tareas del proceso de IS, así como también se muestra su origen correspondiente.

\begin{tabular}{ll}
\hline Nombre & Origen \\
\hline Enunciado del Trabajo & $\cdot$ Cliente \\
\hline Plan del Proyecto Gestión de Proyectos & $\cdot$ Plan del Proyecto Gestión de Proyectos \\
\hline Repositorio del Proyecto Gestión de Proyectos & $\cdot$ Repositorio del Proyecto Gestión de Proyectos \\
\hline
\end{tabular}

Tabla 4 - Productos de entrada IS.

En la Tabla 5, se muestra los productos que la ISO/IEC 29110 señala como aquellos que se generan al realizar las tareas del proceso de IS, así como también se muestra su destino correspondiente. 


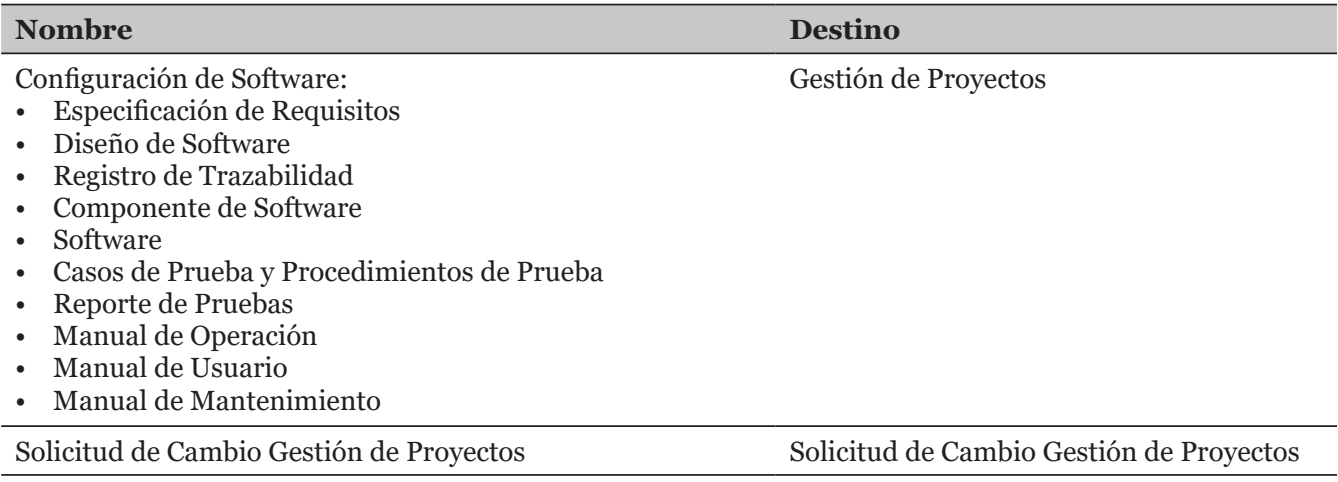

Tabla 5 - Productos de salida IS.

\section{Propuesta}

Para identificar las herramientas necesarias como soporte en la implementacion de la Norma ISO/IEC 29110, en el siguiente apartado, se muestra la propuesta de ciclo de desarrollo, con base en un análisis de la misma norma.

\subsection{Ciclo de Desarrollo}

Se analizaron las actividades y tareas de la ISO/IEC 29110, como resultado se observa un flujo de trabajo combinado. En la Figura 1, se muestra el ciclo de desarrollo propuesto a partir del análisis realizado.

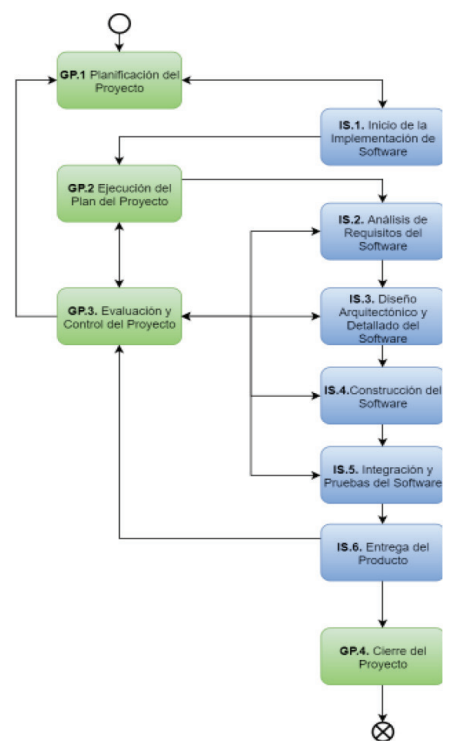

Figura 1 - Propuesta de ciclo de desarrollo. 
Como puede observarse en la Figura 1, el ciclo inicia en GP.1 Planificación del Proyecto, en donde se documenta los detalles de la planificación necesarios para la gestión del proyecto.

Seguido de GP.1, le sigue la actividad IS.1 Inicio de la Implementación de Software, en donde se asegura que el Plan de Proyecto establecido en la actividad anterior, es comprendido por el equipo de desarrollo y se establece el ambiente de trabajo. De existir algún desacuerdo identificado por el equipo de trabajo, se puede regresar a la actividad anterior para la modificación o adaptación del plan de proyecto.

La siguiente actividad es GP.2. Ejecución del Plan del Proyecto, en donde se implementa el plan documentado en el proyecto, esta actividad inicia la actividad IS.2. Análisis de Requisitos del Software, donde se analiza los requisitos acordados con el Cliente y establece los requisitos del proyecto validados.

Desde la actividad IS.2, le sigue de manera secuencial las actividades IS.3. Diseño Arquitectónico y Detallado del Software, donde se transforman los requisitos del Software en la arquitectura de software del sistema y en el diseño detallado del Software; IS.4. Construcción del Software, donde se desarrolla el código y datos del Software a partir del Diseño de Software; IS.5. Integración y Pruebas del Software, donde se asegura que los componentes de software integrados satisfacen los requisitos del software.

Las actividades IS.2, IS.3, IS.4 e IS.5 son monitoreadas en paralelo por la actividad GP.3. Evaluación y Control del Proyecto, para evaluar el desempeño del plan contra los compromisos documentados. En caso de existir algún cambio aprobado, GP.3 regresa a GP.1 para realizar las modificaciones necesarias en el plan de trabajo. En caso de que el cambio no afecte el plan de trabajo, regresa a GP.2 para seguir con la secuencia de actividades de IS (IS.2, IS.3, IS.4 e IS.5). De no existir algún cambio, simplemente regresa a monitorear la actividad de IS en que se quedó.

Al llegar a la actividad IS.6. Entrega del Producto, se integra el producto de software para el Cliente. Ya que el desarrollo de este producto ha sido monitoreado desde el inicio de su construcción hasta este punto, en esta actividad se hace la entrega del producto al Cliente de acuerdo con las instrucciones de entrega. De existir alguna irregularidad con el producto, se regresa a GP.3 a evaluar y hacer los cambios correspondientes, en caso contrario se pasa a la actividad GP.4. Cierre del Proyecto, donde se documenta los detalles de planificación necesarios para gestionar el proyecto.

Establecido el ciclo de desarrollo propuesto y la interacción de las actividades tanto de GP como de IS, es necesario dar soporte con herramientas para llevar a cabo algunas de las actividades y tareas en ambos procesos de esta norma.

\subsection{Herramientas}

Las herramientas son una parte importante de cualquier proceso de desarrollo de software. Permiten la automatización, y por lo tanto, una mejor adaptación del proceso entre los miembros del equipo de desarrollo. Por ello, es importante definir y estandarizar entre el equipo, aquellas herramientas que permitan la mejor adaptación de trabajo que implica el proceso de desarrollo de software. 
Para proponer las herramientas que ayuden a cada tarea en las actividades que conforman el proceso de GP e IS, se analizaron cada una de las tareas y la relación que tienen con los productos de entrada, internos y salida que se generan. Como resultado de este análisis se identificaron las siguientes herramientas.

- Podio: Es una herramienta para la gestión de tareas y proyectos, dispone de una versión gratuita de hasta 10 usuarios. Con esa herramienta se pueden organizar reuniones, llamadas o emails para tener controlado los proyectos. Cada proyecto hace referencia a un cliente, así que es fácil buscar conversaciones y resultados anteriores relacionados con un cliente actual, además se puede integrar con otras herramientas como Google Drive, Dropbox, OneDrive, Google Calendar, Excel, Microsoft Exchange (Podio, 2020).

- Suite de Google: ofrece de manera gratuita diferentes servicios de oficina que podrían ser de utilidad en el proceso de implementación de la ISO/IEC 29110, tales como el correo electrónico, docs, slides, hojas de cálculo, formularios, servicio de almacenamiento, y más.

- Documentos Google: es un procesador de textos online que permite crear documentos, darles formato y edición en tiempo real por parte de los colaboradores registrados. Google Docs cuenta también con un historial de versiones ordenadas por fecha y por autor del cambio realizado (Google Docs, 202).

- Hojas de cálculo Google: es un tipo de procesador de documentos que permiten la manipulación de datos dispuestos en formas de tablas. Google permite, mediante su arquitectura basada en la nube, la colaboración entre las personas del equipo a través de la edición en tiempo real (Google Suite, 2020).

- Draw.io: es una herramienta que facilita la elaboración de diagramas en línea. Draw.io tiene una interfaz que es bastante sencilla y fácil de usar, además dispone de una gran variedad deformas y diseños predeterminados que pueden ser modelados de acuerdo con la estandarización de diagramas seleccionada (Chrome web store, 2020).

- Drive: es un sistema de almacenamiento en la nube, que además integra funciones colaborativas en Microsoft Office, permitiendo crear, almacenar y compartir cualquier documento. Actualmente Google Drive ofrece, en su versión gratuita, 15 GB de almacenamiento (Google Drive, 2020).

- GitLab: es una aplicación para todo el ciclo de vida del desarrollo de software. Desde la planificación del proyecto y la gestión del código fuente hasta la Configuración de Integración/Despliegue Continuo (CI/CD), la supervisión y la seguridad. GitLab es una única aplicación para todo el ciclo de vida de DevOps (GitLab, 2020).

- Git: es un sistema de control de versiones distribuido gratuito y de código abierto diseñado para manejar todo, desde proyectos pequeños hasta muy grandes, con velocidad y eficiencia. Git es fácil de aprender y tiene una huella pequeña con un rendimiento increíblemente rápido . Supera las herramientas de SCM como Subversion, CVS, Perforce y ClearCase con características como ramificación local barata, áreas de preparación convenientes y múltiples flujos de trabajo (Git, 2019). 
- Jest es un framework de prueba de JavaScript, con un enfoque en la simplicidad. Funciona con proyectos utilizando: Babel, TypeScript, Node, React, Angular y Vue. El objetivo de Jest es trabajar fuera de la caja, configurando libremente en la mayoría de los proyectos de JavaScript (Jest, 2020.

Cabe mencionar que como resultado del analisis de las tareas y las herramientas antes descritas, algunos productos de salida deben de combinarse al momento de elaborar un producto de entrada, interno o de salida.

\subsubsection{Herramientas para el Proceso GP}

Tras el análisis del conjunto de las 26 tareas que se indican en el proceso GP, las herramientas propuestas para este proceso son: Google documento (Doc), Google Hoja de cálculo (HC), Google Drive (D),Podio (), Git (G).

A continuación, se muestran en la Tabla 6 a la 9, las tareas (columna 1) de las cuatro actividades respectivamente, que son cubiertas por las herramientas propuestas (columnas $3-7$ ) con una " $\mathrm{X}$ " $\mathrm{y}$ que en algunas tareas se deben complementar las diferentes herramientas que se proponen.

La actividad GP 1. Planificación del Proyecto. La Tabla 6, muestra las herramientas que cubren las 15 tareas, las cuales son:

\begin{tabular}{|c|c|c|c|c|c|c|}
\hline \multirow{2}{*}{ Tarea } & \multirow{2}{*}{ Producto } & \multicolumn{3}{|c|}{ Google } & \multirow{2}{*}{$\mathbf{P}$} & \multirow{2}{*}{$\mathbf{G}$} \\
\hline & & Doc & HC & $\mathbf{D}$ & & \\
\hline GP.1.1 Revisar al Enunciado de Trabajo. & Enunciado de trabajo & $\mathrm{X}$ & & & & \\
\hline $\begin{array}{l}\text { GP.1.2 Definir con el cliente las instrucciones de } \\
\text { entrega. }\end{array}$ & $\begin{array}{l}\text { Enunciado de trabajo/ } \\
\text { Plan de proyecto. }\end{array}$ & $\mathrm{X}$ & & & & \\
\hline GP.1.3 Identificar las tareas especificas a realizar. & $\begin{array}{l}\text { Enunciado de trabajo/ } \\
\text { Plan de proyecto. }\end{array}$ & & & & $\mathrm{X}$ & \\
\hline $\begin{array}{l}\text { GP.1.4 Establecer la duración estimada para realizar } \\
\text { cada tarea. }\end{array}$ & Plan de proyecto & & & & $\mathrm{X}$ & \\
\hline GP.1.5 Identificar y documentar los recursos. & $\begin{array}{l}\text { Enunciado de trabajo/ } \\
\text { Plan de proyecto. }\end{array}$ & $\mathrm{X}$ & $\mathrm{X}$ & & & \\
\hline $\begin{array}{l}\text { GP.1.6 Establecer la composición del equipo de } \\
\text { trabajo. }\end{array}$ & Plan de Proyecto & & & & $\mathrm{X}$ & \\
\hline $\begin{array}{l}\text { GP.1.7 Asignar las fechas de inicio y fin estimadas } \\
\text { para cada tarea. }\end{array}$ & Plan de proyecto & & & & $\mathrm{X}$ & \\
\hline $\begin{array}{l}\text { GP.1.8 Calcular y documentar el esfuerzo y costo } \\
\text { estimado del proyecto. }\end{array}$ & Plan de proyecto & & $\mathrm{X}$ & & $\mathrm{X}$ & \\
\hline GP.1.9 Identificar y documentar los riesgos. & Plan de proyecto & & $\mathrm{X}$ & & & \\
\hline $\begin{array}{l}\text { GP.1.10 Documentar la estrategia de control } \\
\text { de versiones. }\end{array}$ & $\begin{array}{l}\text { Enunciado de trabajo/ } \\
\text { plan de proyecto }\end{array}$ & $\mathrm{X}$ & & & & $\mathrm{X}$ \\
\hline $\begin{array}{l}\text { GP.1.11 Generar el plan de proyecto integrando los } \\
\text { elementos identificados. }\end{array}$ & $\begin{array}{l}\text { Enunciado de trabajo/ } \\
\text { plan de proyecto }\end{array}$ & $\mathrm{X}$ & & & & \\
\hline
\end{tabular}




\begin{tabular}{lllll}
\hline Tarea & Producto & \multicolumn{2}{c}{ Google } \\
\cline { 2 - 4 } & Doc & HC & D & G \\
\hline $\begin{array}{l}\text { GP.1.12 Incluir la descripción del producto, alcance, } \\
\text { objetivos y entregables en el plan de proyecto. }\end{array}$ & $\begin{array}{l}\text { Enunciado de trabajo/ } \\
\text { plan de proyecto }\end{array}$ & $\mathrm{X}$ & \\
\hline $\begin{array}{l}\text { GP.1.13 Verificar y obtener aprobación del plan del } \\
\text { proyecto. }\end{array}$ & $\begin{array}{l}\text { Plan de proyecto/ } \\
\text { resultados de } \\
\text { verificación. }\end{array}$ & $\mathrm{X}$ & \\
\hline $\begin{array}{l}\text { GP.1.14 Revisar y obtener la aprobación del plan del } \\
\text { proyecto. }\end{array}$ & $\begin{array}{l}\text { Plan de proyecto/Acta } \\
\text { de reunión. }\end{array}$ & $\mathrm{X}$ & \\
\hline $\begin{array}{l}\text { GP.1.15 Establecer el repositorio del proyecto } \\
\text { usando la estrategia de control de versiones. }\end{array}$ & $\begin{array}{l}\text { Plan de proyecto/ } \\
\text { Repositorio del } \\
\text { proyecto }\end{array}$ & $\mathrm{X}$ & $\mathrm{X}$ \\
\hline
\end{tabular}

Tabla 6 - Propuesta de herramientas para GP.1.

La Actividad 2. Ejecución del proyecto. La Tabla 7, muestra las herramientas que cubren las 6 tareas, las cuales son:

\begin{tabular}{|c|c|c|c|c|c|c|}
\hline \multirow{2}{*}{ Tarea } & \multirow{2}{*}{ Producto } & \multicolumn{3}{|c|}{ Google } & \multirow{2}{*}{$\mathbf{P}$} & \multirow{2}{*}{ G } \\
\hline & & Doc & HC & D & & \\
\hline $\begin{array}{l}\text { GP.2.1 Monitorear la ejecución del Plan } \\
\text { del Proyecto y registrar la información } \\
\text { actual en el Reporte de Avance. }\end{array}$ & $\begin{array}{l}\text { Plan de proyecto/Reporte de } \\
\text { Avance }\end{array}$ & & & & $\mathrm{X}$ & \\
\hline $\begin{array}{l}\text { GP.2.2 Analizar y evaluar el impacto en } \\
\text { costo, tiempo e impacto técnico de la } \\
\text { Solicitud de Cambio. }\end{array}$ & $\begin{array}{l}\text { Solicitud de cambio/plan de } \\
\text { proyecto }\end{array}$ & $\mathrm{X}$ & & $\mathrm{X}$ & $\mathrm{X}$ & \\
\hline $\begin{array}{l}\text { GP.2.3 Conducir reuniones de revisión con } \\
\text { el Equipo de Trabajo. }\end{array}$ & $\begin{array}{l}\text { Plan de proyecto/Reporte de } \\
\text { avance/acciones correctivas/ } \\
\text { acta de reunión }\end{array}$ & $\mathrm{X}$ & & $\mathrm{X}$ & $\mathrm{X}$ & \\
\hline $\begin{array}{l}\text { GP.2.4 Realizar reuniones con el Cliente, } \\
\text { de las cuales se registran acuerdos y se } \\
\text { dará seguimiento hasta su conclusión. }\end{array}$ & $\begin{array}{l}\text { Plan de proyecto/Reporte de } \\
\text { avance/solicitud de cambio/ } \\
\text { correctivas/acta de reunión }\end{array}$ & $\mathrm{X}$ & & $\mathrm{X}$ & $\mathrm{X}$ & \\
\hline $\begin{array}{l}\text { GP.2.5 Realizar el Respaldo del } \\
\text { Repositorio del Proyecto de acuerdo con la } \\
\text { Estrategia de Control de Versiones. }\end{array}$ & $\begin{array}{l}\text { Respaldo del repositorio del } \\
\text { proyecto }\end{array}$ & & & $\mathrm{X}$ & & $\mathrm{X}$ \\
\hline $\begin{array}{l}\text { GP.2.6 Realizar la recuperación del } \\
\text { Repositorio del Proyecto utilizando el } \\
\text { Respaldo del Repositorio del Proyecto, en } \\
\text { caso de ser necesario. }\end{array}$ & $\begin{array}{l}\text { Respaldo del repositorio del } \\
\text { proyecto/Repositorio del } \\
\text { proyecto }\end{array}$ & & & $\mathrm{X}$ & & $\mathrm{X}$ \\
\hline
\end{tabular}

Tabla 7 - Propuesta de herramientas para GP.2.

La Actividad 3. Evaluación y control del proyecto. La Tabla 8, muestra las herramientas que cubren las 3 tareas a desarrollar: 


\begin{tabular}{|c|c|c|c|c|c|c|}
\hline \multirow{2}{*}{ Tarea } & \multirow{2}{*}{ Producto } & \multicolumn{3}{|c|}{ Google } & \multirow{2}{*}{$\mathbf{P}$} & \multirow{2}{*}{ G } \\
\hline & & Doc & HC & D & & \\
\hline $\begin{array}{l}\text { GP.3.1 Evaluar el progreso del proyecto con respecto al } \\
\text { Plan del Proyecto. }\end{array}$ & $\begin{array}{l}\text { Plan de } \\
\text { proyecto/ } \\
\text { Reporte de } \\
\text { Avance }\end{array}$ & & & & $\mathrm{X}$ & \\
\hline $\begin{array}{l}\text { GP.3.2 Establecer acciones para corregir desviaciones } \\
\text { o problemas e identificar riesgos que amenacen el } \\
\text { cumplimiento del plan. }\end{array}$ & $\begin{array}{l}\text { Reporte } \\
\text { de avance/ } \\
\text { acciones } \\
\text { correctivas }\end{array}$ & $\mathrm{X}$ & & & $\mathrm{X}$ & \\
\hline $\begin{array}{l}\text { GP.3.3 Identificar cambios a requisitos y/o al } \\
\text { Plan del Proyecto para hacer frente a desviaciones } \\
\text { importantes, potenciales riesgos o problemas relativos } \\
\text { al cumplimiento del plan. }\end{array}$ & $\begin{array}{l}\text { Reporte } \\
\text { de avance/ } \\
\text { Solicitud de } \\
\text { cambio }\end{array}$ & $\mathrm{X}$ & $\mathrm{X}$ & & $\mathrm{X}$ & \\
\hline
\end{tabular}

Tabla 8 - Propuesta de herramientas para GP.3.

Actividad 4. Cierre del proyecto. La Tabla 9, muestra las herramientas que cubren las 2 tareas a desarrollar:

\begin{tabular}{|c|c|c|c|c|c|c|}
\hline \multirow{2}{*}{ Tarea } & \multirow{2}{*}{ Producto } & \multicolumn{3}{|c|}{ Google } & \multirow{2}{*}{$\mathbf{P}$} & \multirow{2}{*}{$\mathbf{G}$} \\
\hline & & Doc & $\mathrm{HC}$ & $\mathbf{D}$ & & \\
\hline $\begin{array}{l}\text { GP.4.1 Formalizar la conclusión del proyecto de } \\
\text { acuerdo con las Instrucciones de Entrega establecidas } \\
\text { en el Plan del Proyecto. }\end{array}$ & $\begin{array}{l}\text { Plan de proyecto/ } \\
\text { Configuración de } \\
\text { Software/acta de } \\
\text { Aceptación }\end{array}$ & $\mathrm{X}$ & & & & \\
\hline GP.4.2 Actualizar el Repositorio del Proyecto. & $\begin{array}{l}\text { Configuración } \\
\text { de Software/ } \\
\text { Repositorio del } \\
\text { proyecto }\end{array}$ & $\mathrm{X}$ & & $\mathrm{X}$ & & $\mathrm{X}$ \\
\hline
\end{tabular}

Tabla 9 - Propuesta de herramientas para GP.4.

\subsubsection{Herramientas para el Proceso IS}

Tras el análisis del conjunto de las 42 tareas que se indican en el proceso IS, las herramientas propuestas para este proceso son: Documentos (doc), Draw.io (DI), Podio (P), GitLab (GL) y Jest.

A continuación, se muestran en las Tablas de la 10 a la 15, las tareas (columna 1) de las cuatro actividades respectivamente, que son cubiertas por las herramientas propuestas (columnas $3-7$ ) con una " $\mathrm{X}$ " y que en algunas tareas se deben complementar las diferentes herramientas que se proponen.

Actividad IS 1. Inicio de la Implementación de Software. La Tabla 10, muestra las herramientas que cubren las 2 tareas a desarrollar: 


\begin{tabular}{lllll}
\hline \multirow{2}{*}{ Actividad } & Producto & \multicolumn{2}{c}{ Google } & \multirow{2}{*}{ GL Jest } \\
\cline { 3 - 5 } & Doc & DI & & \\
\hline $\begin{array}{l}\text { IS.1.1 Revisar el Plan del Proyecto actual con los } \\
\text { miembros del Equipo de Trabajo. }\end{array}$ & Plan de Proyecto & & $\mathrm{X}$ & \\
\hline $\begin{array}{l}\text { IS.1.2 Establecer o actualizar el ambiente de } \\
\text { implementación. }\end{array}$ & $\begin{array}{l}\text { Plan de proyecto/ } \\
\text { Ambiente de } \\
\text { implementación }\end{array}$ & $\mathrm{X}$ & $\mathrm{X}$ & \\
\hline
\end{tabular}

Tabla 10 - Propuesta de herramientas para IS.1.

Actividad IS 2. Análisis de Requisitos del Software. La Tabla 11, muestra las herramientas que cubren las 7 tareas a desarrollar:

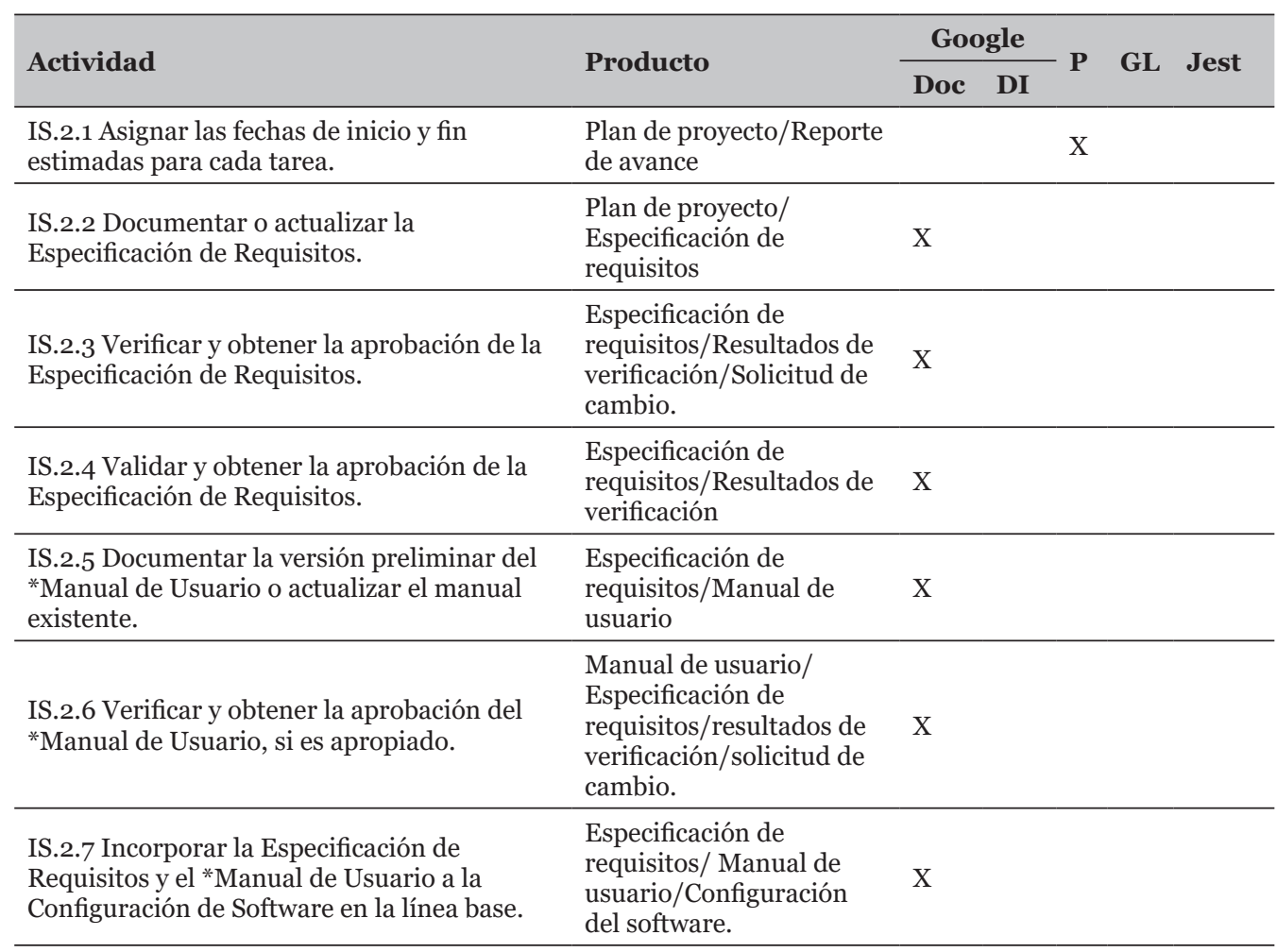

Tabla 11 - Propuesta de herramientas para IS.2.

Actividad IS 3. Arquitectura y Diseño Detallado del Software. La Tabla 12, muestra las herramientas que cubren las 8 tareas a desarrollar: 


\begin{tabular}{|c|c|c|c|c|c|c|}
\hline \multirow{2}{*}{ Actividad } & \multirow{2}{*}{ Producto } & \multicolumn{2}{|c|}{ Google } & \multirow{2}{*}{$\mathbf{P}$} & \multirow{2}{*}{ GL } & \multirow{2}{*}{$\begin{array}{l}\text { jestjs. } \\
\text { io }\end{array}$} \\
\hline & & Doc & DI & & & \\
\hline $\begin{array}{l}\text { IS.3.1 Monitorear la ejecución del Plan } \\
\text { del Proyecto y registrar la información } \\
\text { actual en el Reporte de Avance. }\end{array}$ & Plan de proyecto & & & $\mathrm{X}$ & & \\
\hline $\begin{array}{l}\text { IS.3.2 Comprender la Especificación de } \\
\text { Requisitos. }\end{array}$ & $\begin{array}{l}\text { Especificación de } \\
\text { requisitos }\end{array}$ & $\mathrm{X}$ & & & & \\
\hline $\begin{array}{l}\text { IS.3.3 Documentar o actualizar el Diseño } \\
\text { de Software. }\end{array}$ & $\begin{array}{l}\text { Especificación de } \\
\text { requisitos/Diseño de } \\
\text { software/Registro de } \\
\text { Trazabilidad }\end{array}$ & $\mathrm{X}$ & & & & \\
\hline $\begin{array}{l}\text { IS.3.4 Verificar y obtener la aprobación } \\
\text { del Diseño de Software. }\end{array}$ & $\begin{array}{l}\text { Diseño de software/ } \\
\text { registro de trazabilidad/ } \\
\text { especificación de } \\
\text { requisitos/Resultados de } \\
\text { verificación }\end{array}$ & $\mathrm{X}$ & & & & \\
\hline $\begin{array}{l}\text { IS.3.5 Establecer o actualizar los Casos de } \\
\text { Prueba y Procedimientos de Prueba. }\end{array}$ & $\begin{array}{l}\text { Especificación de } \\
\text { requisitos/diseño } \\
\text { de software/Casos y } \\
\text { procedimientos de prueba }\end{array}$ & $\mathrm{X}$ & & & $\mathrm{X}$ & $\mathrm{X}$ \\
\hline $\begin{array}{l}\text { IS.3.6 Verificar y obtener la aprobación } \\
\text { de los Casos de Prueba y Procedimientos } \\
\text { de Prueba. }\end{array}$ & $\begin{array}{l}\text { Casos y procedimientos } \\
\text { de prueba/Especificación } \\
\text { de requisitos/Resultados } \\
\text { de Verificación/Diseño de } \\
\text { software }\end{array}$ & $\mathrm{X}$ & & & & $\mathrm{X}$ \\
\hline $\begin{array}{l}\text { IS.3.7 Actualizar el Registro de } \\
\text { Trazabilidad incorporando los Casos de } \\
\text { Prueba y Procedimientos de Prueba. }\end{array}$ & $\begin{array}{l}\text { Casos y procedimientos } \\
\text { de prueba/Registro de } \\
\text { trazabilidad }\end{array}$ & $\mathrm{X}$ & & & & $\mathrm{X}$ \\
\hline $\begin{array}{l}\text { IS.3.8 Incorporar el Diseño de Software, } \\
\text { y el Registro de Trazabilidad a la } \\
\text { Configuración de Software como parte de } \\
\text { la línea base. }\end{array}$ & $\begin{array}{l}\text { Diseño de Software/ } \\
\text { Casos y procedimientos } \\
\text { de prueba/Registro de } \\
\text { Trazabilidad/configuración } \\
\text { del software }\end{array}$ & $\mathrm{X}$ & & & $\mathrm{X}$ & $\mathrm{X}$ \\
\hline
\end{tabular}

Tabla 12 - Propuesta de herramientas para IS.3.

Actividad IS 4. Construcción de Software. La Tabla 13, muestra las herramientas que cubren las 7 tareas a desarrollar:

\begin{tabular}{|c|c|c|c|c|c|c|}
\hline \multirow{2}{*}{ Actividad } & \multirow{2}{*}{ Producto } & \multicolumn{2}{|c|}{ Google } & \multirow{2}{*}{$\mathbf{P}$} & \multirow{2}{*}{ GL } & \multirow{2}{*}{ Jest } \\
\hline & & Doc & DI & & & \\
\hline $\begin{array}{l}\text { IS.4.1 Asignar Tareas a los miembros del } \\
\text { Equipo de Trabajo de acuerdo con cada rol, } \\
\text { basado en el Plan del Proyecto actual. }\end{array}$ & Plan de proyecto & & & $\mathrm{X}$ & & \\
\hline IS.4.2 Entender el Diseño de Software. & Diseño de Software & $\mathrm{X}$ & $\mathrm{X}$ & $\mathrm{X}$ & & \\
\hline $\begin{array}{l}\text { IS.4.3 Construir o actualizar los Componente } \\
\text { de Software basados en la parte detallada del } \\
\text { Diseño de Software. }\end{array}$ & $\begin{array}{l}\text { Diseño de software/ } \\
\text { Registro de Trazabilidad/ } \\
\text { componente de software }\end{array}$ & $\mathrm{X}$ & $\mathrm{X}$ & & $\mathrm{X}$ & \\
\hline
\end{tabular}






Tabla 13 - Propuesta de herramientas para IS.4.

Actividad IS 5. Integración y Pruebas del Software. La Tabla 14, muestra las herramientas que cubren las 11 tareas a desarrollar las cuales son:

\begin{tabular}{|c|c|c|c|c|c|c|}
\hline \multirow{2}{*}{ Actividad } & \multirow{2}{*}{ Producto } & \multicolumn{2}{|c|}{ Google } & \multirow{2}{*}{$\mathbf{P}$} & \multirow{2}{*}{ GL } & \multirow{2}{*}{$\begin{array}{l}\text { jestjs. } \\
\text { io }\end{array}$} \\
\hline & & Doc & DI & & & \\
\hline $\begin{array}{l}\text { IS.5.1 Asignar Tareas a los miembros del } \\
\text { Equipo de Trabajo de acuerdo con cada rol, } \\
\text { basado en el Plan del Proyecto actual. }\end{array}$ & Plan de proyecto & & & $\mathrm{X}$ & & \\
\hline $\begin{array}{l}\text { IS.5.2 Entender los Casos de Prueba y } \\
\text { Procedimientos de Prueba. Establecer o } \\
\text { actualizar el ambiente de prueba. }\end{array}$ & $\begin{array}{l}\text { Casos y procedimientos } \\
\text { de prueba/ Ambiente } \\
\text { de prueba }\end{array}$ & $\mathrm{X}$ & & & & $\mathrm{X}$ \\
\hline $\begin{array}{l}\text { IS.5.3 Integrar el Software usando los } \\
\text { Componente de Software y actualizar los } \\
\text { Casos de Prueba y Procedimientos de Prueba } \\
\text { para las pruebas de integración, conforme } \\
\text { sea necesario. }\end{array}$ & $\begin{array}{l}\text { Componente de } \\
\text { software/Casos y } \\
\text { procedimientos de } \\
\text { prueba/registro de } \\
\text { trazabilidad/software }\end{array}$ & $\mathrm{X}$ & & & $\mathrm{X}$ & $\mathrm{X}$ \\
\hline $\begin{array}{l}\text { IS.5.4 Realizar pruebas de Software usando } \\
\text { Casos de Prueba y Procedimientos de } \\
\text { Prueba para la integración y documentar los } \\
\text { resultados en el Reporte de Pruebas. }\end{array}$ & $\begin{array}{l}\text { Software/Casos y } \\
\text { procedimientos de } \\
\text { prueba/Reporte de } \\
\text { pruebas }\end{array}$ & $\mathrm{X}$ & & & $\mathrm{X}$ & $\mathrm{X}$ \\
\hline $\begin{array}{l}\text { IS. } 5 \cdot 5 \text { Corregir los defectos encontrados } \\
\text { y realizar una prueba de regresión hasta } \\
\text { satisfacer el criterio de finalización. }\end{array}$ & $\begin{array}{l}\text { Software/Reporte } \\
\text { de pruebas/Casos y } \\
\text { procedimientos de } \\
\text { prueba/Registro de } \\
\text { trazabilidad }\end{array}$ & $\mathrm{X}$ & & & $\mathrm{X}$ & $\mathrm{X}$ \\
\hline $\begin{array}{l}\text { IS.5.6 Actualizar el Registro de Trazabilidad } \\
\text { en caso de ser necesario. }\end{array}$ & $\begin{array}{l}\text { Software/Registro de } \\
\text { trazabilidad/ }\end{array}$ & $\mathrm{X}$ & & & $\mathrm{X}$ & \\
\hline $\begin{array}{l}\text { IS.5.7 Documentar el *Manual de Operación } \\
\text { o actualizar el manual actual, en caso de ser } \\
\text { apropiado. }\end{array}$ & $\begin{array}{l}\text { Software/Manual de } \\
\text { operación }\end{array}$ & $\mathrm{X}$ & & & & \\
\hline
\end{tabular}




\begin{tabular}{|c|c|c|c|c|c|c|}
\hline \multirow{2}{*}{ Actividad } & \multirow{2}{*}{ Producto } & \multicolumn{2}{|c|}{ Google } & \multirow{2}{*}{$\mathbf{P}$} & \multirow{2}{*}{ GL } & \multirow{2}{*}{$\begin{array}{l}\text { jestjs. } \\
\text { io }\end{array}$} \\
\hline & & Doc & DI & & & \\
\hline $\begin{array}{l}\text { IS.5.8. Verificar y obtener la aprobación } \\
\text { del *Manual de Operación, en caso de ser } \\
\text { necesario. }\end{array}$ & $\begin{array}{l}\text { Manual de Operación/ } \\
\text { Software/Resultados de } \\
\text { verificación }\end{array}$ & $\mathrm{X}$ & & & $\mathrm{X}$ & \\
\hline $\begin{array}{l}\text { IS.5.9 Documentar el * Manual de Usuario o } \\
\text { actualizar el actual, si aplica. }\end{array}$ & $\begin{array}{l}\text { Software/Manual de } \\
\text { Usuario }\end{array}$ & $\mathrm{X}$ & & & $\mathrm{X}$ & \\
\hline $\begin{array}{l}\text { IS.5.10 Verificar y obtener la aprobación } \\
\text { del *Manual de Usuario, en caso de ser } \\
\text { necesario. }\end{array}$ & $\begin{array}{l}\text { Software/Manual de } \\
\text { usuario/resultados de } \\
\text { verificación }\end{array}$ & $\mathrm{X}$ & & & & \\
\hline $\begin{array}{l}\text { IS.5.11 Incorporar los Casos de Prueba } \\
\text { y Procedimientos de Prueba, Software, } \\
\text { Registro de Trazabilidad, Reporte de } \\
\text { Pruebas, *Manual de Operación y *Manual } \\
\text { de Usuario a la Configuración de Software } \\
\text { como parte de la línea base. }\end{array}$ & $\begin{array}{l}\text { Casos y procedimientos } \\
\text { de prueba/Software/ } \\
\text { Reporte de pruebas/ } \\
\text { Registro de } \\
\text { trazabilidad/Manual de } \\
\text { operación/Manual de } \\
\text { Usuario/Configuración } \\
\text { de software }\end{array}$ & $\mathrm{X}$ & & & $\mathrm{X}$ & $\mathrm{X}$ \\
\hline
\end{tabular}

Tabla 14 - Propuesta de herramientas para IS.5.

Actividad IS 6. Entrega del Producto. La Tabla 15, muestras las herramientas que cubren las 8 tareas a desarrollar:

\begin{tabular}{|c|c|c|c|c|c|c|}
\hline \multirow{2}{*}{ Actividad } & \multirow{2}{*}{ Producto } & \multicolumn{2}{|c|}{ Google } & \multirow{2}{*}{$\mathbf{P}$} & \multirow{2}{*}{ GL } & \multirow{2}{*}{ Jest } \\
\hline & & Docs & DI & & & \\
\hline $\begin{array}{l}\text { IS.6.1 Asignar Tareas a los } \\
\text { miembros del Equipo de Trabajo } \\
\text { de acuerdo con cada rol, basado } \\
\text { en el Plan del Proyecto actual. }\end{array}$ & Plan de proyecto & & & $\mathrm{X}$ & & \\
\hline $\begin{array}{l}\text { IS.6.2 Comprender la } \\
\text { Configuración de Software. }\end{array}$ & Configuración del software & $\mathrm{X}$ & & & $\mathrm{X}$ & \\
\hline $\begin{array}{l}\text { IS.6.3 Documentar el Manual de } \\
\text { Mantenimiento o actualizar el } \\
\text { actual. }\end{array}$ & $\begin{array}{l}\text { Configuración del Software/ } \\
\text { Manual de Mantenimiento }\end{array}$ & $\mathrm{X}$ & & & $\mathrm{X}$ & \\
\hline $\begin{array}{l}\text { IS.6.4 Verificar y obtener la } \\
\text { aprobación del Manual de } \\
\text { Mantenimiento. }\end{array}$ & $\begin{array}{l}\text { Manual de mantenimiento/ } \\
\text { Configuración del software/ } \\
\text { Resultado de verificación }\end{array}$ & $\mathrm{X}$ & & & $\mathrm{X}$ & \\
\hline $\begin{array}{l}\text { IS.6.5 Incorporar el Manual de } \\
\text { Mantenimiento a la línea base de } \\
\text { la Configuración de Software. }\end{array}$ & $\begin{array}{l}\text { Configuración del software/ } \\
\text { Manual de Mantenimiento }\end{array}$ & $\mathrm{X}$ & & & $\mathrm{X}$ & \\
\hline $\begin{array}{l}\text { IS.6.6 Llevar a cabo la entrega de } \\
\text { acuerdo con las Instrucciones de } \\
\text { Entrega. }\end{array}$ & $\begin{array}{l}\text { Plan de proyecto/ } \\
\text { Configuración del software }\end{array}$ & $\mathrm{X}$ & & $\mathrm{X}$ & $\mathrm{X}$ & \\
\hline
\end{tabular}

Tabla 15 - Propuesta de herramientas para IS.6. 


\subsubsection{Cobertura global de Herramientas para el proceso GP e IS.}

En la Tabla 16, se muestra el resumen de las tareas cubiertas por actividad por parte de las herramientas (columnas 3 - 10). En la última fila se encuentra el porcentaje de la cobertura por parte de la herramienta, teniendo como base las 68 (100\%) tareas de los dos proceso.

\begin{tabular}{|c|c|c|c|c|c|c|c|c|c|}
\hline \multirow[b]{2}{*}{ Actividad } & \multirow[b]{2}{*}{ Tareas } & \multicolumn{4}{|c|}{ Google } & \multirow[b]{2}{*}{ Podio } & \multirow[b]{2}{*}{ GitLab } & \multirow[b]{2}{*}{ Git } & \multirow[b]{2}{*}{ Jest } \\
\hline & & Docs & $\begin{array}{l}\text { Hoja de } \\
\text { Calculo }\end{array}$ & Drive & Draow.io & & & & \\
\hline GP. 1. & 15 & 8 & 3 & 1 & o & 5 & o & 2 & o \\
\hline GP. 2. & 6 & 3 & o & 5 & o & 4 & o & 2 & o \\
\hline GP. 3. & 3 & 2 & 1 & o & o & 3 & o & o & o \\
\hline GP. 4. & 2 & 2 & o & 1 & o & 0 & 0 & 1 & 0 \\
\hline IS. 1. & 2 & 1 & o & o & o & 2 & o & o & o \\
\hline IS. 2. & 7 & 6 & o & o & o & 1 & o & 0 & 0 \\
\hline IS. 3. & 8 & 7 & o & o & o & 1 & 2 & o & 4 \\
\hline IS. 4. & 8 & 4 & o & o & 2 & 2 & 5 & o & 2 \\
\hline IS. 5. & 11 & 10 & o & o & o & 1 & 7 & o & 5 \\
\hline IS. 6. & 6 & 5 & o & o & o & 2 & 5 & o & o \\
\hline Total & 68 & 48 & 4 & 7 & 2 & 21 & 19 & 5 & 11 \\
\hline$\%$ & & 70.59 & 5.88 & 10.29 & 2.94 & 30.88 & 27.94 & $7 \cdot 35$ & 16.18 \\
\hline
\end{tabular}

Tabla 16 - Resumen de cobertura de herramientas propuestas para GP e IS

Con respecto a las actividades de GP, puede observarse, en la Tabla 16, la herramienta que cubre mas tareas de las 4 actividades de este proceso es la suite de google teniendo en primer lugar: Documentos, Drive, Hoja de Calculo y Draow.io, sin embargo, en esta herramienta se deben definir formatos que permitan automatizar las tareas. En este contexto, la herramienta con campos definidos y que tiene una cobertura amplia es Podio para definir estimaciones de tiempo y que ayudan a dar seguimiento al proyecto.

Con respecto a las actividades de IS, puede observarse en la Tabla 16, la herramienta que cubre mas tareas de las 6 actividades del proceso IS es nuevamente la suite de google, siendo el uso de documentos el que mas incidencia tiene. Sin embargo, el proceso de IS esta enfocado a la Implementacion y/o desarrollo del software por lo que las herramientas que mas incidencia tienen durante el desarrollo de activiades relacionadas al desarrollo son: Podio, GitLab, Jest, este ultimo da soporte para el establecimiento de pruebas que solicita la norma.

\section{Estudio de caso}

Tomando como base un cuestionario guía para la extracción del conocimiento de una organización, tanto para GP como para IS, se realizaron entrevistas a miembros del 
centro de desarrollo del Instituto de Tecnológico de Loreto, Zacatecas, México de los tres niveles básicos: operativo, administrativo y gerencial. De esta manera se entrevistaron a dos desarrolladores y un líder técnico.

De cada entrevista se obtuvieron diagramas para GP e IS. El gestor de proyecto proporcionó sus diagramas de manera directa, cumpliendo así con la diagramación de los tres niveles básicos de una organización.

De esta manera, se obtuvieron diagramas de cada una de las entrevistas realizadas y se validaron los procesos identificados con cada entrevistado.

Tomando los diagramas como base, se analizaron y se validaron con cada una de las cuatro personas entrevistadas, para verificar que la información proporcionada concuerda con su forma de visualizar su trabajo dentro del equipo de desarrollo.

Una vez que los entrevistados aprobaron sus respectivos diagramas de forma de trabajo, se realizó una matriz para identificar las actividades en común del equipo de trabajo y visualizar aquellas actividades diferentes entre ellos. En la Figura 2, se muestra la primera parte de la matriz realizada.

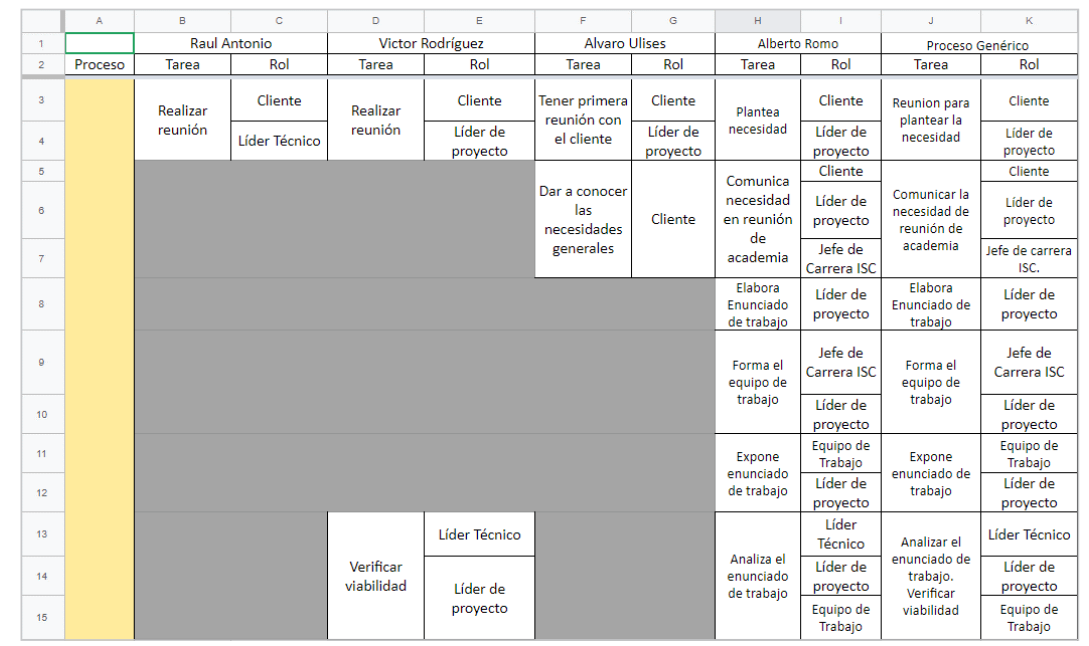

Figura 2 - Matriz de trazabilidad de actividades entre entrevistas.

Tomando como base la Matriz de trazabilidad delas actividades, se realizóla diagramación de los procesos GP e IS, identificados a partir de las actividades mencionadas en las entrevistas anteriores a los miembros del equipo de trabajo del centro de desarrollo de software del ITSLoreto. Una vez que se han identificado los procesos genéricos de GP e IS del centro de desarrollo del ITSLoreto, se identificaron aquellas tareas de ISO/IEC 29110 que son cubiertas por los procesos genéricos de ITSLoreto (Figura 3).

La Figura 3, muestra a manera de ejemplo la trazabilidad de las actividades identificadas hacia la norma. 
Una vez realizada la trazabilidad por parte de la organización de sus procesos hacia los procesos de la norma ISO/IEC 29110, la empresa pudo identificar que herramientas le daban soporte para la realizacion de sus tareas orientadas a esta norma. Asimismo, esta organización detecto que un punto critico es la automatizacion de las pruebas que se estaban realizando de manera manual y con muy poca informacion para realizar acciones correctivas durante el desarrollo del software.

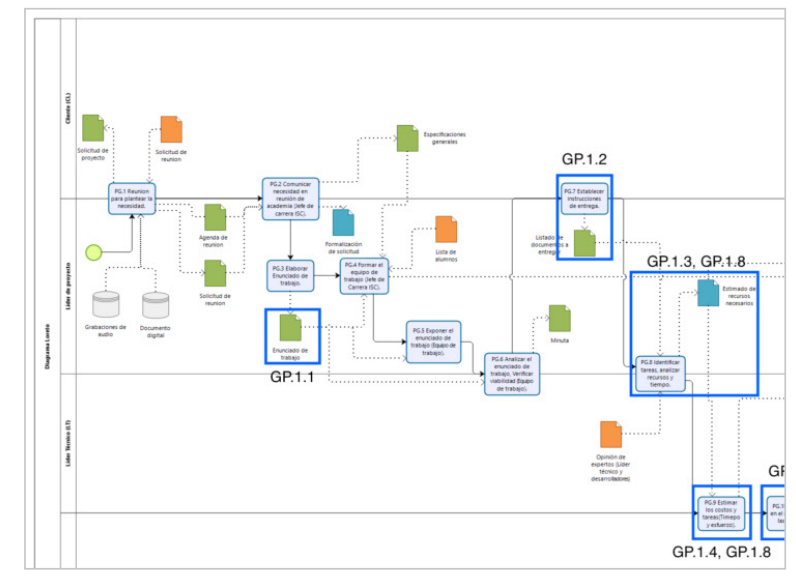

Figura 3 - Trazabilidad de proceso genérico con ISO/IEC 29110 (ejemplo).

\section{Conclusiones}

Gracias a la observación del estudio de caso, se logró detectar dos de las principales complicaciones que presentan las organizaciones al implementar por primera vez el estándar ISO/IEC 29110:

- La resistencia al cambio en su forma de trabajar, aun más, si una norma como la ISO/IEC 29110 no tiene un soporte de herramientas que la acompañen para la implementación de algunas actividades o tareas.

- No conocen los términos ni los entregables que requiere la ISO/IEC 29110. Al facilitar y familiarizarse con las herramientas de manera inferencial, se familiarizan con la terminologia y los entregables que deben cumplir de acuerdo a la norma.

- Automatizacion de pruebas, un punto alarmante fue identificar que este tipo de organizaciones desconoce como establecer procesos, procedimiento y tecnicas de pruebas, en este sentido, la herramienta Jest da un soporte a las pruebas que espefica esta norma.

El trabajo presentado propone una guia de herramientas que dan soporte a las tareas de los procesos de GP e IS para desarrollar los productos de Entrada, Internos y de Salida. Con base en la trazabilidad presentada entre sus procesos y los de la norma, la organización pueda identificar que herramientas pueden ser utilizadas para desarrollar los entregables que pide la norma ISO/IEC 29110 e iniciar una mejora continua. 
Como trabajo futuro se esta proponiendo realizar una herramientas global que pueda gestionar la creación de formatos y poder automatizar la captura y generación de entregables de acuerdo a los productos indicados en los procesos de GP e IS de la Norma ISO/IEC 29110.

\section{Agradecimientos}

Este trabajo se pudo culminar gracias al trabajo previo y apoyo del equipo de trabajo conformado por la Dra. Mirna Muñoz, y los alumnos: Patricia Montoya-Méndez, Héctor Girón-Bobadilla, Isaac Rodríguez-Maldonado y de la participación del Centro de Desarrollo del Instituto Tecnológico Superior de Loreto (ITSLoreto), perteneciente al Ecosistema de Calidad en Tics del Estado de Zacatecas, México.

\section{Referencias}

Chrome web store. (2020). [En línea]. Available: https://chrome.google.com/webstore/ detail/drawio-diagrams/onlkggianjhjenigcpigpjehhpplldkc.

Git. (2020). [En línea]. Available: https://git-scm.com/. (Accessed: 23- Mar- 2020).

GitLab. (2020). [En línea]. Available: https://about.gitlab.com.

Google Docs. (2020). [En línea]. Available: https://www.google.com/intl/es_mx/docs/ about/\#start.

Google Drive. (2020). [En línea]. Available: https://www.google.com/intl/es-419_ $\mathrm{mx} /$ drive/.

Google Suite. (2020). [En línea]. Available: https://gsuite.google.com.mx/intl/es-419_ $\mathrm{mx} /$ products/sheets/.

INEGI. (2020). Resultados de la encuesta nacional sobre productividad y competitividad de las micro, pequeñas y medianas empresas (enaproce) 2018. [Online]. Available: https://www.inegi.org.mx/contenidos/saladeprensa/boletines/2019/especiales/ ENAPROCE2018.pdf. (Accessed: 23- Mar- 2020).

ISO/IEC 29110. (2012). Perfiles del ciclo de vida para las pequeñas organizaciones (PO). Parte 5-1-2: Guía de gestión e ingeniería: Grupo de perfil genérico, Perfil básico, 2012. Lima: INDECOPI.

ISO/IEC. (2018). Systems and software engineering - Lifecycle profiles for Very Small Entities (VSEs) - Part 4-1: Software engineering - Profile specifications: Generic profile group [En línea]. Available: https://www.iso.org/obp/ui/\#iso:std:isoiec:29110:-4-1:ed-2:v1:en.

Jest. (2020). [En línea] https://jestjs.io/

NYCE. (2020). Certificación, Verificación, Normalización. [Online]. Available: https://www.nyce.org.mx/. (Accessed: 23- Mar- 2020). 
O'Connor, R., \& Laporte, C. (2017). The Evolution of the ISO/IEC 29110 Set of Standards and Guides. International Journal of Information Technologies and Systems Approach, 10(1), 1-21.

Podio. (2020). Project Management and Collaboration Software, Podio, 2020. [Online]. Available: https://podio.com/site/?gclid=CjwKCAiA7t3yBRADEiwA4GFlI9YfFm lonoqzwwauyV2nj8_j-R95jENJq1-2Ee8xF4AKCIfyAYOYDxoCXrsQAvD_BwE. (Accessed: 23- Mar- 2020).

Project Management Institute. (2013). Guía de los Fundamentos para la Dirección de Proyectos PMBOK, PMI, Quinta Edición. Newtown Square: Project Management Institute. 